Tomas AF, Sánchez, RJ, Pizzi H, Pizzi RD, Dib MD, Paez Rearte MG, Pizzi HL.

Cátedra de Parasitología y Micología Médicas FCM, UNC.
Trabajo recibido: 03 de marzo 2016. Aprobado: 04 abril 2016.

\section{ENTORNO ECOLÓGICO DEL CASO ÍNDICE DE LEISHMANIASIS CUTÁNEA, UNQUILLO, CÓRDOBA}

ENVIRONMENTAL SETTING OF THE INDEX CASE OF SKIN LEISHMANIASIS, UNQUILLO, CORDOBA

ENTORNO ECOLÓGICO DO CASO ÍNDICE LEISHMANIOSE TEGUMENTAR, UNQUILLO, CÓRDOBA

\section{Resumen}

INTRODUCCIÓN Y OBJETIVOS: Las Sierras de Córdoba se encuentran al OESTE de la provincia y se disponen en tres cordones, en uno de éstos por su vertiente ESTE, se ubica la localidad de Unquillo, lugar del primer caso autóctono de leishmaniasis cutánea en la provincia de Córdoba, Argentina, el cual ha sido reportado y publicado por nosotros (1). Creemos oportuno relevar los factores ecológicos y demográficos que pueden haber favorecido en la radicación de Lutzomya spp. MATERIALES Y MÉTODOS: Inspección in situ y relevamiento observacional del casco urbano y periférico de la localidad. Análisis bibliográfico de estadísticas, censos, geomorfología y fitogeografía para caracterizar el patrón que sirve de nicho ecológico al vector. RESULTADOS: Unquillo tiene una geografía de faldeos, fracturas y barrancos, con suelos ricos en nutrientes orgánicos, buenos niveles pluviométricos y parches de actividad humana en el ambiente silvestre que resultan un conjunto ideal para la cría y reproducción del insecto transmisor. CONCLUSIONES.: El estudio muestra lo que denominados corredores de escurrimiento de Lutzomya spp., convergentes desde provincias endémicas con la zoonosis hacia las localidades serranas, 
entre éstas, Unquillo. El análisis arroja un valor extra y una amenaza para la salud pública, si se tiene en cuenta la cercanía de la localidad con la gran urbe de la ciudad de Córdoba.

Palabras clave: leishmaniasis cutánea, Lutzomya, Unquillo, Córdoba

\section{Abstract}

INTRODUCTION AND OBJECTIVES: Cordoba hills are located to the WEST of the province and are arranged in three mountain ranges, in one of these, on the EASTERN slope, we find the town of Unquillo, place of the first autonomous case of skin leishmaniasis in the province of Cordoba, Argentina, which was reported and published by us (1).We consider important to gather information about environmental and demographic factors that may have favored the settlement of Lutzomya spp. MATERIALS AND METHODS: On-site inspection, observational examination of the city and surrounding areas, bibliographic analysis of statistics, census, geomorphology and phytogeography were used to characterize the pattern which functions as the ecological niche for the vector.RESULTS: The geography of Unquillo presents slopes, faults and ravines, with soil rich in organic nutrients, good levels of rainfalls and human activity patches in the wild environment resulting in the ideal setting for the settlement and reproduction of the transmitting insect. CONCLUSIONS: The study shows the so-called draining corridors of Lutzomya spp., convergent from endemic provinces with the zoonosis towards these mountain tows; Unquillo among them. The analysis adds extra value and a threat for public health if we consider the proximity of this town to the large city of Cordoba.

Key Words: skin leishmaniasis, Lutzomya, Unquillo, Cordoba

\section{Resumo}

INTRODUÇÃO E OBJETIVOS: As serras de Córdoba estão localizadas ao OESTE da província e são organizadas em três cordões de montanhas, estando sediada em um deles, na sua encosta LESTE, a cidade de Unquillo, local do primeiro caso autóctone de leishmaniose cutânea na província de Córdoba, Argentina, que tem sido relatado e publicado por nós (1). Achamos oportuno fazer um levantamento dos fatores ecológicos e demográficos que podem ter favorecido o estabelecimento de Lutzomya spp. MATERIAIS E MÉTODOS: Inspeção in situ e pesquisa observacional da área urbana e periférica da cidade. Análise bibliográfica de estatísticas, recenseamento, geomorfologia e fitogeografia para caracterizar o padrão que serve de nicho ecológico ao vetor. RESULTADOS: Unquillo tem uma geografia de encostas, fraturas e barrancos, com solo rico em nutrientes orgânicos, bons níveis de precipitação e manchas de atividade humana na natureza que são ideais para criação e reprodução do inseto transmissor. CONCLUSÕES:. O estudo mostra que os chamados corredores de escoamento de Lutzomya spp, convergindo de províncias com zoonose endêmica para as cidades de montanha, entre elas, Unquillo. A análise produz um valor extra e uma ameaça para a saúde pública, levando em conta a proximidade deste povoado para o grande centro urbano da cidade de Córdoba.

Palavras-chave: Leishmaniose cutânea, Lutzomya, Unquillo, Córdoba

\section{Introducción}

Las Sierras de Córdoba están localizadas al OESTE de la provincia y se disponen en tres cordones en dirección norte-sur separados por valles. Uno de éstos está conformado por las Sierras Chicas, al pie de las mismas y por su vertiente ESTE, se ubica la localidad de Unquillo, lugar de procedencia del primer caso autóctono de leishmaniasis cutánea en la provincia de Córdoba, Argentina, el cual ha sido registrado y recientemente publicado por 
nuestro equipo de trabajo (1). La leishmaniasis en sus distintas formas clínicas representa un grave problema de salud en el mundo que merece atenderse de modo prioritario. Para que la emergencia de la zoonosis se produzca, es necesaria la presencia de un pequeño insecto del género Lutzomya spp.; que es el vector de un protozoario: Leishmania spp. (2). Creemos indispensable y oportuno relevar las condiciones y todo el orden de factores ecológicos y demográficos que pueden haber favorecido en la reciente radicación de Lutzomya spp. Este hecho adquiere una importancia añadida ya que Unquillo dista 30 km de la ciudad de Córdoba, la cual tiene 1.329.604 habitantes (6). La leishmaniasis está vinculada a un medio que permite la supervivencia del vector, así se reconocen factores propios del hombre, la presencia de animales domésticos, ganado, los desplazamientos migracionales, las variables climáticas y el sistema de gestión de residuos. La Lutzomya spp. se adapta al hábitat humano y su peri domicilio. Las deficiencias de los sistemas de recolección de residuos y los vertederos a cielo abierto, promueven el desarrollo de los lugares de cría, reposo de los vectores y aumentan su acceso a la población humana. (2, 3).

\section{Objetivo:}

Estudiar las variables ecológicas que inciden en la radicación del vector, hecho que se infiere por la emergencia del primer caso testigo.

\section{Materiales y Método}

Se realizó una evaluación ecológica de Unquillo, a través de la observación e inspección ocular del casco urbano, periférico de la localidad, cubriendo todos los relieves a través de varias jornadas. Se realizaron los recorridos de manera aleatoria para el análisis observacional de campo. En segundo término se efectuó la búsqueda bibliográfica para determinar el conjunto ecológico característico de la localidad, determinando los factores geomorfológicos, fitogeográficos, climatológicos, demográficos y revisando las áreas endémicas de leishmaniasis en nuestro país.

\section{Resultados}

A la observación y relevación en terreno se examina que los faldeos orientales de las Sierras Chicas, soportan la degradación ambiental y patrones de desarrollo urbanísticos anárquicos y con graves carencias de infraestructura sanitaria y disposición de residuos. En las localidades de las Sierras Chicas, se observan parches del bosque serrano típico entre los emprendimientos edilicios y económicos. Los asentamientos humanos en barrios sin planificación, la presencia de animales en torno al domicilio, una geografía de bosque chaqueño con lugares sombreados y rocas salientes sirven de refugio y cría de vectores.

Tienen estas localidades escasa agresión química, lo que favorece la reproducción de vectores transmisores en contraste con las llanuras del ESTE y el exterminio de especies por los agroquímicos. La actividad ganadera pequeña, suma a suelos orgánicos ricos en nutrientes vegetales, los orgánicos animales ideales para el desarrollo del insecto (10).

La caracterización geomorfológica dicta que la provincia de Córdoba se ubica en la región central de Argentina en las coordenadas $29^{\circ} 30^{\prime}-35^{\circ} 00^{\prime}$ latitud sur (LS) y $61^{\circ} 45^{\prime}$ $65^{\circ} 45^{\prime}$ longitud oeste (LO), con una superficie de $165.321 \mathrm{~km} 2$ (6).

Unquillo en el departamento Colón, se ubica en los 31¹3'60”' LS y 64¹9’00” LO.

Hay dos tipos de relieve: sierras y planicies. Estas últimas son las que más degradación han sufrido por la acción del hombre y el modelo de sobreexplotación agrícola.

El cordón de las Sierras Chicas se extiende en dirección N/NE-S/SO desde el cerro El Pajarillo al norte hasta las márgenes del Río Tercero al Sur. La vertiente occidental es abrupta y áspera, mientras que la oriental presenta pendientes suaves que descienden 


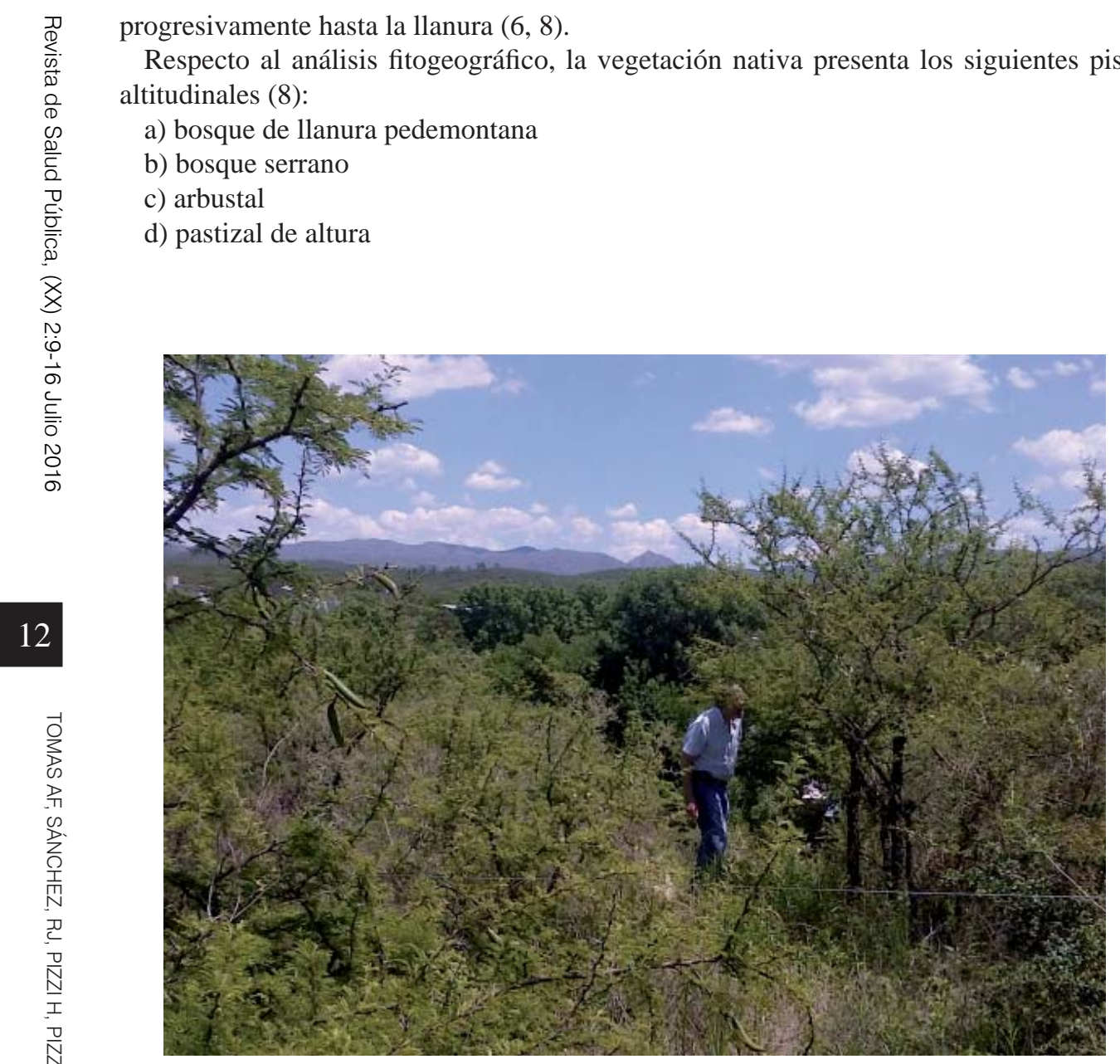

Fig. 1. 2014. Contexto ecoepidemiológico de Unquillo, Córdoba, Argentina Fuente: Fotografía propia. Cátedra de Parasitología y Micología Médicas. FCM. UNC

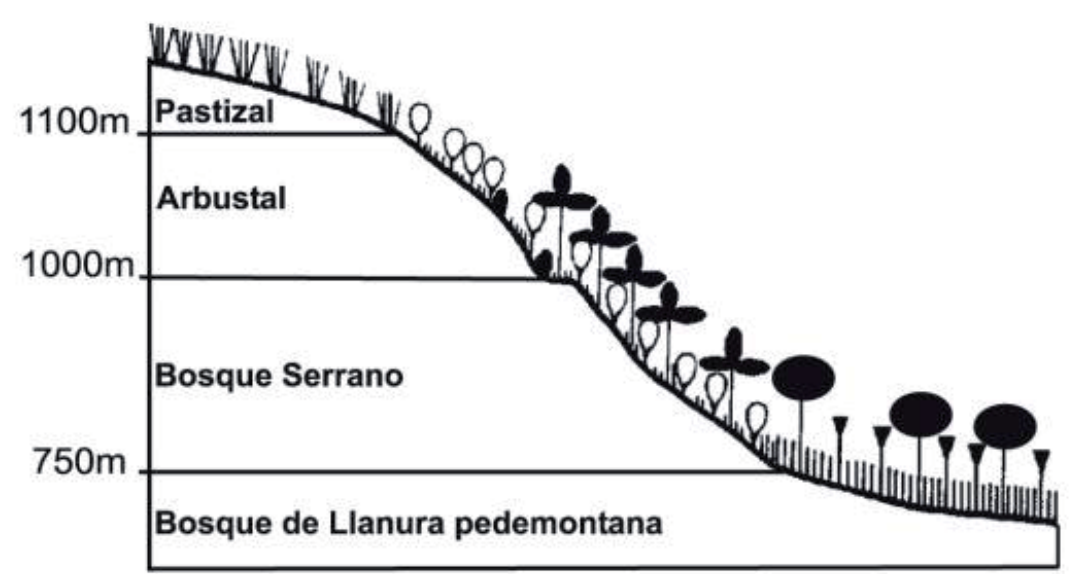

Fig. 2:Perfil de la distribución altitudinal de la vegetación nativa en las Sierras Chicas según Kurtz (1904), Luti et al. (1979), Estrabou (1983) y Cabido \& Zak (1999) en Gavier \& Bucher (2004). 
A su vez, la llanura chaqueña comprende la llanura extra serrana oriental, que se extiende desde el Norte de la provincia por el faldeo oriental de las Sierras del Norte a los 500 metros sobre nivel del mar; hacia el Este hasta la depresión del Mar de Ansenuza y hacia el Sur, coincidiendo con un límite edáfico difuso y se encuentra entre los $29^{\circ} 35^{\prime}$ y $31^{\circ} 10^{\prime}$ de LS y los $63^{\circ} 25^{\prime}$ a los $64^{\circ} 25^{\prime}$ de LO. Cubre una superficie aproximada de 10.000 $\operatorname{km} 2(6,8)$.

El bosque de llanura pedemontana se ubica por debajo de los 750 metros sobre nivel del mar, y es dominado por el quebracho blanco (Aspidosperma, quebracho blanco).En su mayor parte ha sido degradado a bosques secundarios o espinillares por acción antrópica, con un marcado incremento de espinillo (Acaciacaven), aromo (A. atramentaria), garabato (A.praecox) y algarrobo (Prosopis spp.) (8).

Desde la llanura chaqueña al Este de la región ascienden especies maderables constituyendo un pedemonte ecotonal ondulado. La fauna silvestre es rica dado su variado origen (subtropical, andino-patagónica y pampeano) y la diversidad de biotopos $(6,8)$.

En cuanto al plano climático, las Sierras Chicas están sujeta al tipo semiárido templado con régimen continental monzónico, de precipitaciones concentradas entre octubre y marzo, -media anual de 780 mm.- En la ladera oriental -Unquillo-, las lluvias son más abundantes (960 mm anuales) y disminuyen de Este a Oeste. La topografía montañosa influye en la existencia de microclimas y creación de nichos ecológicos específicos para el asentamiento de vectores. El promedio de precipitación anual promedió en los últimos 40 años es de $949 \mathrm{~mm}$ y la temperatura media de $18,9^{\circ} \mathrm{C}$, con una máxima y una mínima absolutas de $40,1 \mathrm{y}-6,6{ }^{\circ} \mathrm{C}$, respectivamente (9).

Según el Censo Nacional 2010, el departamento Colón tiene 224.487 habitantes y 76.420 viviendas. El crecimiento poblacional del departamento ha sido vertiginoso en las últimas dos décadas, ya que fue de 36,4\% entre 1991 y 2001, y de 31,2 \% entre 2001 y 2010. La población registrada en el Censo 2010 fue de 225.151 habitantes con una densidad de $86,9 \mathrm{hab} / \mathrm{km} 2$

El área endémica de leishmaniasis, en la Argentina, ocupa una superficie aproximada de 500 mil km2 en 10 provincias, que desde el Oeste al Este son: Jujuy, Salta, Tucumán, Catamarca, Santiago de Estero, Formosa, Chaco, Misiones, Corrientes y Santa Fe. Las que abarcan 7 ecoregiones: Yungas, Chaco seco, Chaco húmedo, Selva Paranaense, Esteros del Iberá, campos, malezas, y espinal (12).

Si bien el límite sur aproximado de esta área está definido hasta los $28^{\circ} 29^{\prime} \mathrm{LS}$, las citas de captura de vectores competentes abarcan un área superior llegando hasta los $31^{\circ} 35^{\text {' }}$ LS $(12,14)$.

La ecología, estacionalidad y comportamiento del vector determinan las características de los ciclos de transmisión, existiendo una fuerte relación entre la bioregión que ocupan y el tipo de respuesta epidémica que producen $(12,14)$.

Es necesario considerar que en áreas recientemente deforestadas la presencia de vectores se vio modificada, pudiendo provocar un aumento de contacto vector-hombre, así como una buena adaptación de algunas especies de vectores a ambientes peridomiciliarios (14, $15)$.

La distribución de las Lutzomyasen en el espacio es heterogénea, con áreas de alta abundancia de vectores que se entremezclan con otras de baja o nula abundancia, coherente con las predicciones de transmisores principales o "core transmitters" de Woolhouse, et al. Estas áreas son dinámicas en el espacio y en el tiempo, y se ven definidas principalmente por las características micro-ambientales del lugar $(17,18)$. 


\section{Conclusiones}

La localidad de Unquillo, con faldeos, fracturas, barrancos, rocas expuestas, suelos ricos, variables térmicas y pluviométricas que acompañan, resumen un nicho ecológico propicio para el establecimiento del vector de la leishmaniasis.

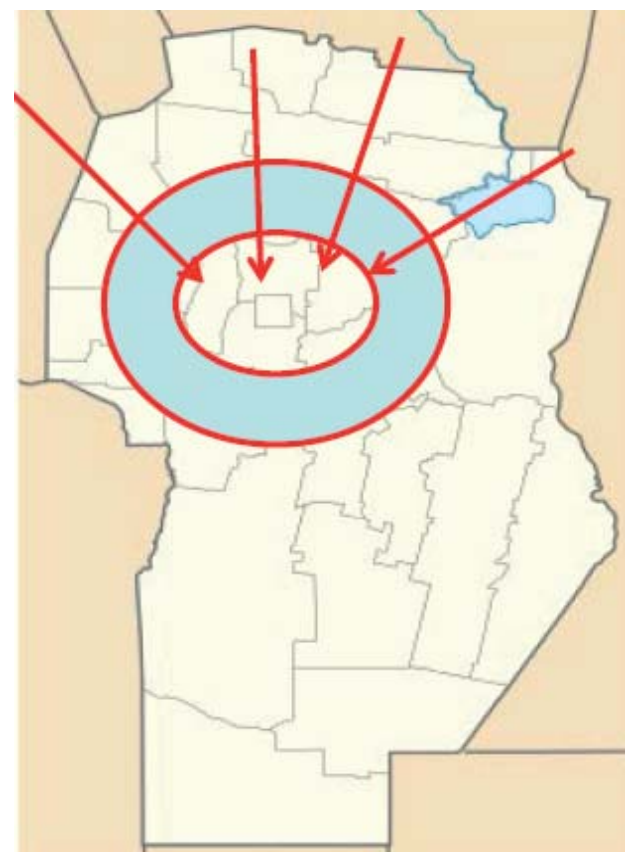

Figura 3. Corredores de escurrimiento del vector de leishmaniasis desde Catamarca, Santiago del Estero, Santa Fe hacia las Sierras Chicas provincia de Córdoba. Elaboración propia sobre mapa de la provincia de Córdoba. Año 2014 Cátedra de Parasitología y Micología Médicas.FCM UNC

El relevamiento fitogeográfico (bolsón chaqueño) muestra la existencia de corredores de escurrimiento del insecto, que ingresan desde Catamarca, Santiago del Estero y la Laguna de Ansenuza para converger y alcanzar los faldeos orientales de las Sierra Chicas, que ofrece las condiciones ambientales, demográficas y fitogeográficas para la radicación y permanencia del vector.

Tal como señala Kufner y Tamburini (22), la vertiente oriental de las Sierra Chicas de la provincia de Córdoba (Argentina), destaca por el atractivo paisajístico que incentivó el avance sin regulación de la urbanización y procesos conexos sobre el pedemonte y las laderas, generando impactos y conflictos socio-ambientales, en desmedro de su calidad ambiental y potencialidad de desarrollo (23) y con las consecuencias sanitarias descriptas.

La zona se muestra como una alternativa para el asentamiento humano por las cercanías con la ciudad de Córdoba y la geografía serrana característica. De tal manera, la localidades de las Sierras Chicas muestran un crecimiento demográfico importante, así en 1991 contaban con 109.489 h, en 2001, 172.179 y para 2010 con 225.151 h. (6).

Otra circunstancia que favorece la migración de vectores hacia la zona es el calentamiento global. En la zona oriental de las Sierras Chicas hubo cambios notorios de las clásicas isohietas, isobaras e isotermas. Esta situación se potencia con la desproporcionada deforestación, aumento poblacional y la falta de planificación edilicia.

La ecoepidemiología requiere diferenciar causa de casos y causa de incidencia, así como estudiar los eventos de salud a multiescala de tiempo y espacio (Susser) (24). En ese sentido a macroescala (tendencias macroeconómicas), la generación de los brotes y dispersión de leishmaniasis cutánea, en los últimos 50 años se asocia a variación climática, ligada a cambios en el uso de la tierra, mientras la dispersión reciente de leishamaniasis 
en su variante visceral, a migración de personas y mascotas desde la zona endémica a la urbanización no planificada y con pobres servicios sanitarios. En cuanto a los vectores es necesario tener en cuenta que las intervenciones con el sólo uso de insecticidas mostraron poca efectividad, permitiendo realizar bloqueos focales de cobertura reducida y de corta duración (19).

Los actuales tiempos imponen a los decisores nuevas obligaciones frente a la sociedad. La preservación del medio ambiente y el manejo integral de los recursos naturales, es uno de los mayores desafíos en la agenda de la dirigencia de los responsables en Salud.

La leishmaniasis está vinculada a un contexto macro sostenido por la tropicalización del clima que ha permitido el avance de la frontera epidemiológica de la zoonosis que toca latitudes cada vez más australes; permitiendo nuevas radicaciones del vector, que se integra en ambientes ecoepidemiológicos cuya definición ha sido el interés del presente trabajo, teniendo en cuenta el reporte del primer caso clínico y la necesidad de instrumentar las políticas sanitarias acordes de manera urgente y prioritaria.La realidad de la zoonosis implica un desafío sanitario de proporciones a la luz de la escasa distancia a la ciudad de Córdoba y sus 1.329.604 habitantes (6).

\section{Bibliografía}

1. Pizzi HL, Tomas AF, Ferrero MR, Pizzi HL(h), Fernández GL, Furey F, Pizzi RD, Herrero M, Dib MD. El inexorable avance de la leishmaniasis: comunicación del primer caso autóctono en la provincia de Córdoba. Revista de Salud Pública, 2015. (XIX). Pág. 15-23.

2. Pizzi HL, Sánchez RJ, Huck GA; Artrópodos. Su impacto en la salud. Córdoba, Argentina. Edit. Rotagraf. 2007. Pág.117-18.

3. Pizzi HL, Sánchez RJ, Huck GA, Tomas AF; Artrópodos. Calentamiento global. Nuevos desafíos. Control de plagas. Córdoba, Argentina. Edit. Rotagraf. 2012. Pág. 139-141.

4. Nirich Ronga S. Regiones Naturales de la Provincia de Córdoba. AGENCIA CÓRDOBA D.A.C.yT, Dirección de Ambiente, 2003. Disponible en: En:http:// www.igualdadycalidadcba.gov.ar/SIPEC CBA/publicaciones/proyarboles/ RegionesNaturalesdeCordoba.pdf

5. Pizzi HL, Sánchez RJ, Huck GA. Protozoología. Córdoba, Argentina. Edit. Rotagraf, 2012. Pág. 45-55.

6. Dirección General de Estadística y Censos de la Provincia de Córdoba. Censo Nacional 2010. Disponible en: http://estadistica.cba.gov.ar/.

7. Instituto Nacional de Parasitología Dr. Mario Fatala Chaben. [Internet] CABA. 2015. [Citado 5 de Mayo 2015]. Leishmaniasis. Disponible en: http://www.anlis. gov.ar/ inp/?page_id=316 , 7. OMS. Leishmaniasis. [Internet] [Citado 5 de Mayo 2015]. Nota descriptiva N³75. Disponible en: http://www.who.int/mediacentre/ factsheets/fs375/es/).

8. Capitanelli J. a. Geomorfología. En: Vázquez JB, Miatello RA \& ME Roqué (Directores). Geografía Física de la Provincia de Córdoba. Banco de la Provincia de Córdoba. Editorial Boldt, Buenos Aires, Argentina.1979. Pág.144-203

9. Capitanelli J. b. Clima. En: Vázquez JB, Miatello RA \& ME Roqué (Directores). Geografía Física de la Provincia de Córdoba. Banco de la Provincia de Córdoba. Editorial Boldt, Buenos Aires, Argentina.1979. Pág. 213-296

10. Gavier GI, Bucher EH: Deforestación de las Sierras Chicas de Córdoba (Argentina) en el período 1970-1997. Academia Nacional de Ciencias,Córdoba, Rep. Argentina. 2004. Miscelánea No 101.

11. SIAN (Sistema Información Ambiental Nacional) http://www.ambiente.gov.ar.

12. Salomon OD, Quintana MG, Mastrangelo AV, Fernández MS. Leishmaniasis 
and Climate Change. Case Study: Argentina Journal Tropical Medicine:2012 Pág.1-12.

13. Salomón OD, Orellano PW, Quintana MG, Pérez S, Sosa Estani S, Acardi SA, Lamfri M. 2006. Transmisión de leishmaniasis tegumentaria en la Argentina. Medicina (Buenos Aires) 66. Pág. 211-219.

14. Salomón OD. Phlebotominae - Flebótomos. Editor. Artrópodos de interés médico en Argentina. Buenos Aires: Fundación Mundo Sano; Publicación Monográfica. 2005. 6. Pág.67-73.

15. Walsh JF, Molyneux DH, Birley MH. Deforestation: effects on vector-borne disease. Parasitology.1993. 106. Pág.55-75.

16. Ashford R.Leishmaniasis reservoirs and their significance in control. Clinical Dermatology. 1996.14. Pág.523-532.

17. Lainson R. Demographic changes and their influence on the epidemiology of the American leishmaniases, in $\mathrm{M}$. W. Service Eds. Demography and vector-borne diseases. CRC Press, Boca Raton, Fla. 1989. Pág.85-106.

18. Woolhouse ME, Dye C, Etard JF, Smith T, Charlwood JD, Garnett GP, et al. Heterogeneities in the transmission of infectious agents: implications for the design of control programs. Proceedings of the National Academy of Sciences (USA). 1997. Pág. 338-342.

19. Santini MS, Fernández MS, Pérez AA, Sandoval AE, Salomón OD.Lutzomyia longipalpis abundance in the city of Posadas, northeastern Argentina: variations at different spatial scales. Memorias do Instituto Oswaldo Cruz, Rio de Janeiro.2012. Pág.106-7.

20. Santini MS, Salomón OD, Acardi SA, Sandoval EA, Tartaglino LC.Lutzomyia longipalpis behavior at an urban visceral leishmaniasis focus in Argentina. Rev. do Instituto de Medicina Tropical de São Paulo.2010.52. Pág. 187-191.

21. Salomón OD. Mesa redonda: Infecciones emergentes y re-emergentes en un mundo globalizado.XVI Jornadas Argentinas de Microbiología y III Congreso Bioquímico del Litoral.Santa Fe, Argentina, 5, 6 y 7 de Agosto de 2015.

22. Tamburini D, Kufner M. Procesos ecológicos y sociales de ocupación del espacio en la Sierra Chica de Córdoba, Argentina. Gestión Ambiental (Chile) 2006.12. Pág. 4154.

23. Moiso A. Determinantes de la Salud. En: Fundamentos de epidemiología. Cap 6. La Plata. 2007. Pág. 161-188. Disponible en: http://www.inus.org.ar/documentacion/ DocumentosTecnicos. 